\title{
Chemical Evolution of the Carina Dwarf Spheroidal
}

\author{
Kate Pilkington* \\ University of Central Lancashire \\ E-mail: kpilkington@uclan.ac.uk
}

\section{Brad K. Gibson}

University of Central Lancashire

E-mail: bkgibson@uclan.ac.uk

\begin{abstract}
We explore a range of chemical evolution models for the Local Group dwarf spheroidal (dSph) galaxy, Carina. A novel aspect of our work is the removal of the star formation history (SFH) as a 'free parameter' in the modeling, making use, instead, of its colour-magnitude diagram (CMD)constrained SFH. By varying the relative roles of galactic winds, re-accretion, and ram-pressure stripping within the modeling, we converge on a favoured scenario which emphasises the respective roles of winds and re-accretion. While our model is successful in recovering most elemental abundance patterns, comparable success is not found for all the neutron capture elements. Neglecting the effects of stripping results in predicted gas fractions approximately two orders of magnitude too high, relative to that observed.
\end{abstract}

XII International Symposium on Nuclei in the Cosmos

August 5-12, 2012

Cairns, Australia

${ }^{*}$ Speaker. 


\section{Introduction}

Local Group dwarf galaxies are critical training sets for all aspects of Galactic Archaeology and high-redshift galactic chemical evolution. They are the only systems in the Universe for which the otherwise highly uncertain (essentially unconstrained) star formation history (SFH) is not an unknown. If one cannot demonstrate an ability to model the chemical evolution of such constrained systems, it limits one's confidence in the ability to do so for (the many more distant and numerous) unresolved stellar populations. We present models of the Local Group dwarf spheroidal (dSph) Carina, as a case study in our probe of the efficacy of galactic chemical evolution. We focus on the use of CMD-derived star formation histories [2] coupled to our chemical evolution package GEtool [3]. We sample a range of inflow and outflow parameterisations, concentrating on Carina due to the unprecedented quality of its spectroscopic data [11]. Carina shows three main sequence turn-offs in its CMD, corresponding to the stellar populations associated with distinct star formation episodes (Fig. 1), the majority of which belong to the intermediate-age burst.

GEtool tracks the time evolution of 107 isotopes (spread over 45 elements); as in our preliminary work on the Sculptor dwarf [4], we employ SNeII yields [12], SNeIa yields [5], and post-processed neutron-capture yields from AGB stars [9]. The underlying yields from low- and intermediate-mass stars has been updated, to reflect recent developments in the field [6]. GEtool allows consideration of feedback from supernova, outflowing galactic winds, parameterised rampressure stripping, re-accretion of stripped/wind ejecta, and infall of fresh fuel for future generations of star formation. We adopt a Kroupa, Tout \& Gilmore [8] initial mass function (IMF) for the work described here.

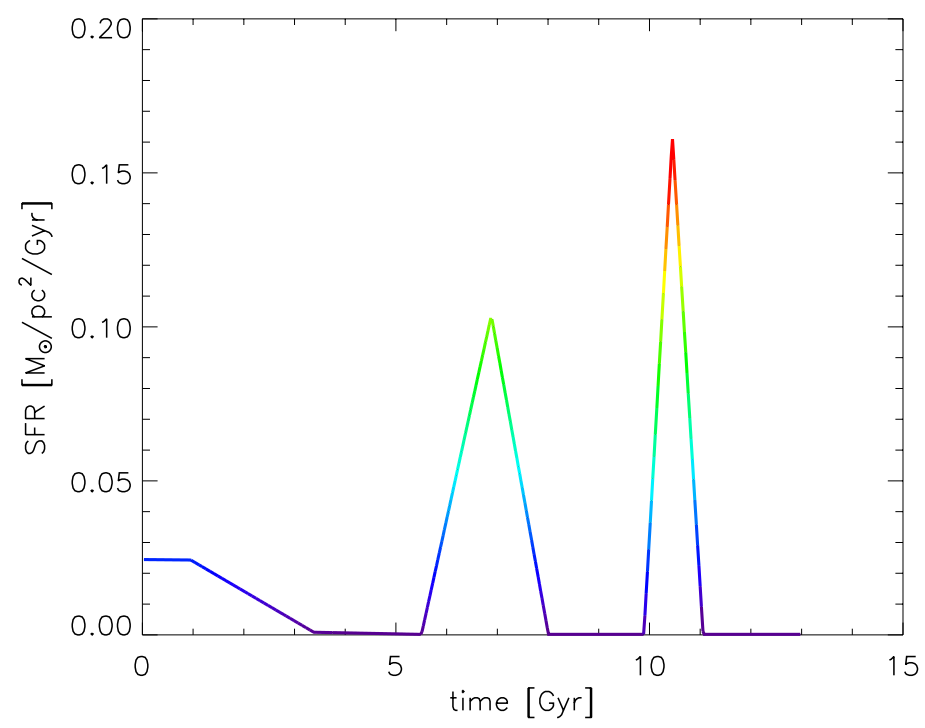

Figure 1: Empirically-derived star formation history of the Carina dwarf spheroidal [2], employed as a 'fixed' input to the chemical evolution model, colour-coded according to star formation rate (see Fig. 3). 


\section{Inflows and Outflows}

We first show the inferred temporal evolution of the total gas surface density of Carina (inset panel within Fig. 2). We can sub-divide this 'total' into four primary sub-components; gas resulting from stellar ejecta (red), that taking part in a galactic wind/outflow (blue), that associated with fresh infall of star formation 'fuel' (purple), and that being 'lost' to star formation at any given time (yellow). The dominant role of gas infall (purple) is readily apparent; in some sense, the primary novel aspect of our modeling is that said infall is parameterised (or 'controlled') to ensure the model adheres strictly to a Kennicutt star formation law of the form $\psi(t)=0.05 \sigma(t)^{1.4} \mathrm{M}_{\odot} \mathrm{pc}^{-2} \mathrm{Gyr}^{-1}$ (where $\psi$ is the star formation rate and $\sigma$ is the gas surface density).

In the absence of exceedingly efficient Type Ia supernovae (SNeIa)-driven outflows and/or ram pressure stripping, the predicted final gas fraction of the model would be $\sim 90 \%$ - i.e., roughly two orders of magnitude higher than observed. Parameterised ram pressure stripping [10] should allow us to better recover the low gas fractions seen in dSphs in the vicinity of massive hosts, like the Milky Way. In the interim, simply stripping the most recently infallen fuel (least tightly bound) provides a suitable final fraction.

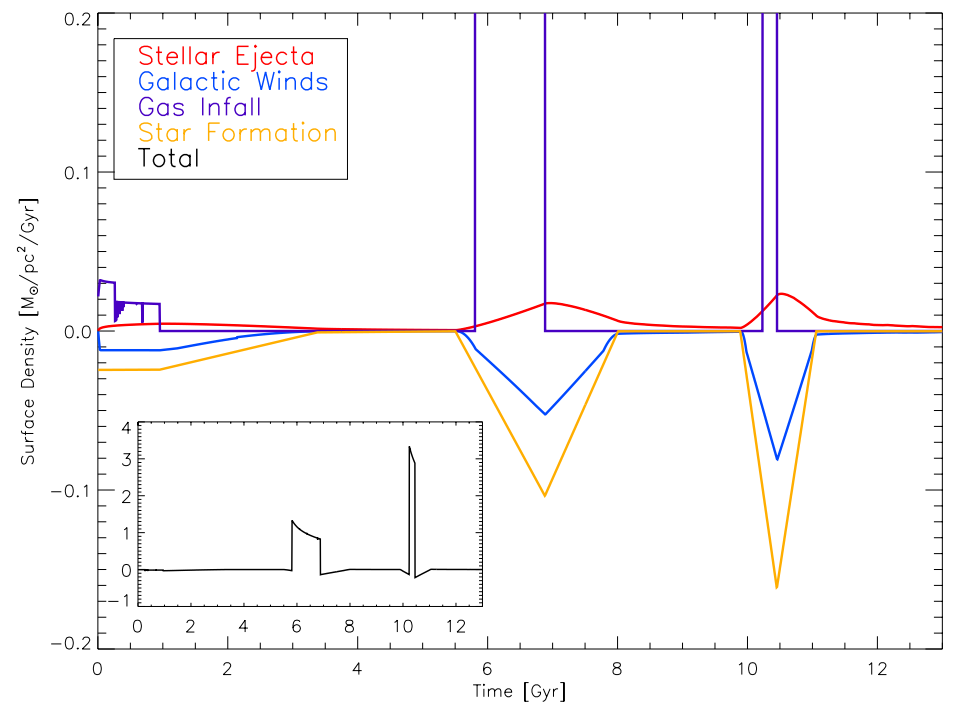

Figure 2: The evolution of the mass surface density of gas. In red is shown the material returned to the system from dying stars, as a function of time; blue represents the amount of gas removed by the galactic winds; yellow represents the amount of gas used up in star formation; purple represents the gas infalling to the system. The inset to the panel shows the sum of the four sources of gas - clearly, infalling material dominates over the other sources ( $\sim 10: 1$ relative to, for example, outflowing wind material).

\section{Abundance Patterns}

\subsection{Alpha Elements}

In Fig 3, we show the abundance patterns of three $\alpha$-elements: magnesium, oxygen, and calcium. We find reasonable agreement with the observational data, particularly for oxygen and 


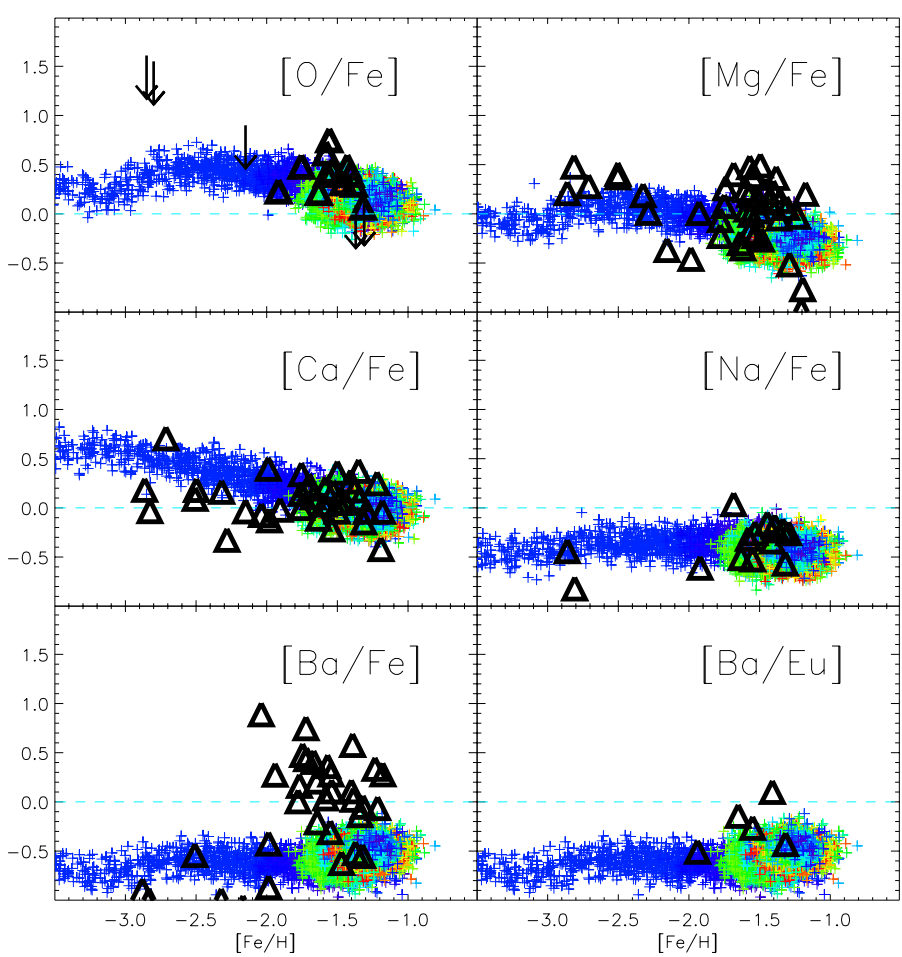

Figure 3: Predicted abundance patterns for the Carina dSph (small dots) coloured according to the star formation rate (recall, Fig 1), where red and green symbols correspond to times of higher star formation rates, while blue and purple correspond to periods of lower star formation. The black triangles correspond to the observational data of Venn et al. [11]; downward facing arrows are also from Venn et al, but represent data for which only upper limits exist.

calcium. Our predictions for magnesium are less ideal, in large part we feel, due to the well-known issue concerning the underproduction of $\mathrm{Mg}$ from the SNeII models of Woosley \& Weaver [12]. Here, we have allowed for re-accreation of outflowing wind material; not doing so, within the context of our framework, leads to an underproduction of the global stellar metallicity. Our earlier models [4] suffered from a significant overproduction of sodium, but as shown clearly in Fig 3, this problem has been rectified naturally via the use of the newer AGB models of Karakas (2010).

\subsection{Neutron Capture Elements: r- and s-process}

Also in Fig 3, we show the predicted distributions of the heavy s-process element barium and the r-process element europium. At low metallicities, we capture the behaviour of the neutron capture elements well, but it is equally clear that we grossly underestimate the dynamic range in both the s- and r-process elemental patterns at higher metallicities.

\subsection{Metallicity Distribution Function (MDF)}

In Fig 4, we show the MDF of our fiducial Carina model (black histogram), alongside the observed MDF from [7] (derived using two different metallicity calibrations: [1], in cyan, and [13] 


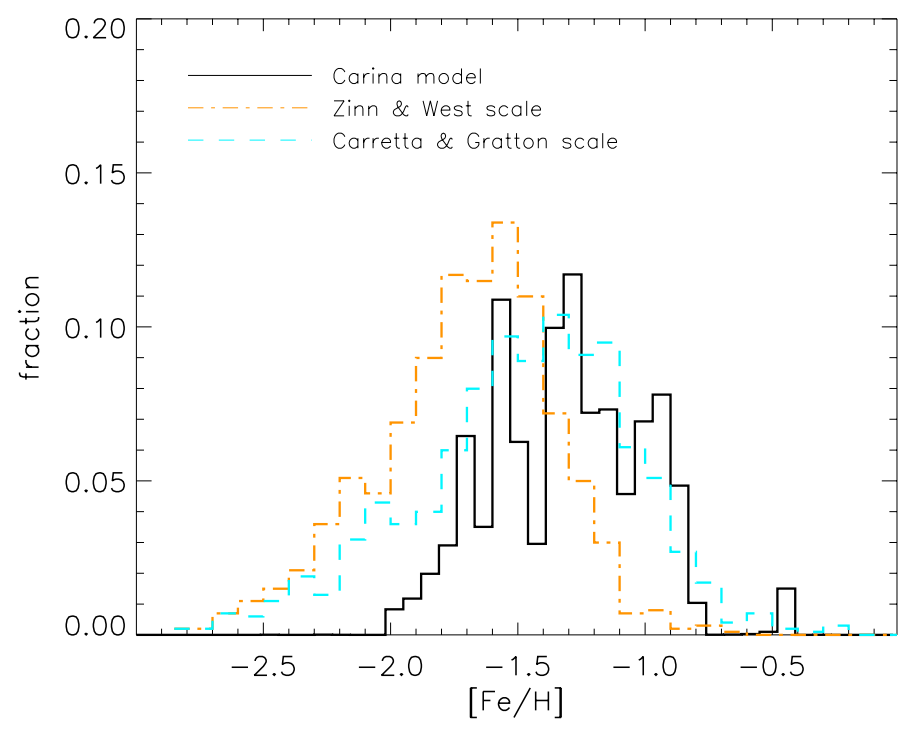

Figure 4: The predicted metallicity distribution function of the Carina dSph (black) compared with the observed MDF of Koch et al. [7] (calibrated with two different metallicity calibrations: [1] in cyan, and [13] in orange). Our model has been convolved with a $\sigma=0.28$ dex Gaussian to mimic the uncertainties associated with observational data.

in orange). The model MDF has been convolved with a $\sigma=0.28$ dex Gaussian, to reflect the quoted observational uncertainties [7]. One can see that the peak of our MDF matches well with the observed peak (cyan), although the current fiducial model admittedly suffers from a dearth of lower metallicity stars in the tail of the MDF.

\section{Conclusions}

1. We show that an infall rate which is recovered by inverting the Kennicutt star formation law $\left(\psi \propto \sigma^{1.4}\right)$ with a CMD-inferred star formation history can successfully match many of the chemical properties of the Carina dSph, without the need for additional 'fine-tuning' of the basic chemical evolution properties.

2. Most elemental abundance patterns are consistent with those observed, save (primarily) for the neutron capture elements.

3. Without invoking some form of parameterised ram pressure stripping, we inevitably overpredict the final gas fraction by two orders of magnitude.

4. Extending our work to the cover the entire sample of Local Group dwarfs with CMD-inferred star formation histories, including samples such as those of LCID (http://http://www.iac.es/proyecto/LCID/), is one of the next steps in this work. 


\section{References}

[1] E. Carretta \& R. Gratton, $A \& A, 121,95$, (1997)

[2] A. E. Dolphin, et al., (2005) [astro-ph/0506430]

[3] Y. Fenner \& B. K Gibson, PASA, 20, 189, (2003)

[4] Y. Fenner, et al., ApJ, 646, 181, (2006)

[5] K. Iwamoto, et al., ApJ, 125, 439, (1999)

[6] A. I Karakas, MNRAS, 403, 1413, (2010)

[7] A. Koch, et al., ApJ, 131, 895, (2006)

[8] P. Kroupa, et al., MNRAS, 292, 545, (1993)

[9] M. Lugaro, et al., ApJ, 586, 1305, (2003)

[10] S. Pasetto, et al., A\&A, 525, A99, (2011)

[11] K. A. Venn, et al., ApJ, 751, 102, (2012)

[12] S. E. Woosley \& T. A. Weaver, ApJ, 101, 181, (1995)

[13] R. Zinn \& M. J. West, ApJ, 55, 45, (1984) 